\title{
Mutual impact of clinically translatable near-infrared dyes on photoacoustic image contrast and in vitro photodynamic therapy efficacy
}

Ljubica Z. Petrovic

Marvin Xavierselvan

Maju Kuriakose

Michael D. Kennedy

Christopher D. Nguyen

Julian J. Batt

Kelsey B. Detels

Srivalleesha Mallidi 


\title{
Mutual impact of clinically translatable near-infrared dyes on photoacoustic image contrast and in vitro photodynamic therapy efficacy
}

\author{
Ljubica Z. Petrovic, ${ }^{\dagger}$ Marvin Xavierselvan, ${ }^{\dagger}$ Maju Kuriakose, ${ }^{\dagger}$ \\ Michael D. Kennedy, Christopher D. Nguyen, Julian J. Batt, \\ Kelsey B. Detels, and Srivalleesha Mallidi* \\ Tufts University, Department of Biomedical Engineering, Medford, Massachusetts, \\ United States
}

\begin{abstract}
Photodynamic therapy (PDT), a spatially localized phototoxic therapy that involves irradiation of a photosensitizer (PS) with specific wavelengths of light, has shown exceptional promise in impacting cancer treatment outcomes, particularly oral cancer. To reduce PDT outcome variability, attempts toward image-guided personalized PDT are being pursued by monitoring PS uptake either via fluorescence or photoacoustic imaging (PAI), a nonionizing modality dependent on optical absorption properties of the tissue. PAI-guided PDT requires a near-infrared contrast agent for deep tissue imaging with minimal photobleaching effect. We evaluate the impact of PDT agent, benzoporphyrin derivative (BPD), on PAI agent indocyanine green (ICG) and vice versa, given that they have different optical absorption properties and singlet oxygen quantum yields for PDT. Specifically, we demonstrate in two oral squamous cell carcinoma lines (FaDu and SCC4) that ICG has minimal effect on BPD PDT efficacy when irradiated with either a continuous or pulsed laser. Furthermore, the impact of BPD on ICG photodegradation was monitored with PAI in tissue-mimicking phantoms. These studies inform us that the combination of BPD and ICG can be utilized for PAI-guided PDT. However, researchers need to consider the photodegradation effects of ICG in the presence of BPD when designing their drug delivery strategies for PAI-guided PDT. (C) The Authors. Published by SPIE under a Creative Commons Attribution 4.0 Unported License. Distribution or reproduction of this work in whole or in part requires full attribution of the original publication, including its DOI. [DOI: 10.1117/1.JBO.25.6 $.063808]$
\end{abstract}

Keywords: photoacoustic imaging; photodynamic therapy; photosensitizer; image-guided therapy; indocyanine green; benzoporphyrin derivative.

Paper 190371SSR received Oct. 18, 2019; accepted for publication Feb. 5, 2020; published online Feb. 28, 2020; corrected Jun. 25, 2020.

\section{Introduction}

The past few decades have seen exponential growth in the utility of light for diagnosis and therapy of cancer. Specifically, in the cancer therapeutics realm, photodynamic therapy (PDT) has shown exceptional promise in impacting outcomes, due to spatially and temporally localized treatment with less systemic toxicity. ${ }^{1-5}$ PDT is a light-based therapy that uses a photosensitizer (PS) to generate cytotoxic reactive molecular species such as the singlet oxygen molecules to target and kill cancer cells. Clinically, PDT's worth as a mainstream therapy is currently being explored for various skin cancers, head and neck cancer, brain tumors, pulmonary and pleural mesothelial cancer, gastroenterological cancer, and urological disease, as well as for oral cancer. ${ }^{6,7}$ Recently, the efficacy of PDT was demonstrated in oral cancer patients with early stage lesions. A single session of PDT treatment resulted in negative biopsies and excellent healing of the mucosa. ${ }^{8}$ Despite promising results, one of the main hurdles for PDT in cancer has been variations in treatment outcome due to intertumor and intratumor heterogeneity in PS uptake due to irregular tumor vascular structure. ${ }^{5,9,10}$ As the PS molecule is inherently a theranostic agent,

\footnotetext{
*Address all correspondence to Srivalleesha Mallidi, E-mail: srivalleesha.mallidi@tufts.edu

†'Eual contribution.
} 
its fluorescence properties have traditionally been utilized to monitor uptake and photobleaching to predict PDT response and reduce the variability in the treatment outcome. ${ }^{11,12}$ However, due to the inability of wide field fluorescence imaging technique to provide three-dimensional images, a comprehensive picture of PS uptake cannot be obtained and there is a need for imaging modalities, such as photoacoustic imaging (PAI), to obtain the heterogeneous distribution of the PS in the tumor.

In PAI, the delivered nanosecond pulsed light energy is absorbed by the dye and converted into heat, leading to thermoelastic expansion and subsequent ultrasound wave emission unlike the radiative relaxation of the excited dye molecule in fluorescence imaging. ${ }^{13-16}$ Primarily, the PAI contrast depends on the optical absorption properties of the dye that enables it to monitor dye uptake $^{17,18}$ and dye-environment interactions. ${ }^{19,20}$ In the near-infrared (NIR) optical window range, indocyanine green (ICG) and IRdye $800 \mathrm{CW}$ are the only U.S. Food and Drug Administration (FDA)-approved dyes for imaging that can provide photoacoustic (PA) contrast. ICG is used as a PA contrast agent either in its free form or associated with different types of nanoparticles. ${ }^{21}$ ICG is not a good PS due to its meager singlet oxygen quantum yield (0.10 to $0.20),{ }^{22,23}$ but it is an excellent contrast agent. On the other hand, benzoporphyrin derivative (BPD) is the only FDA-approved NIR PS with high singlet oxygen quantum yield of $0.77^{24}$ and is not conditioned to be a good PAI agent. Despite being categorized as theranostic agents, neither BPD nor ICG can individually be used successfully for both PDT and PAI simultaneously. Furthermore, the pulsed irradiation utilized for PAI could also be used to excite BPD to generate singlet oxygen and the resultant therapeutic effect is on par with continuous wave $(\mathrm{CW})$ irradiation. ${ }^{25}$ Hence, several avenues are being explored to design entities that can be both excellent PAI and PDT agents, such as conjugating PS with gold nanovesicles that are good PA contrast agents ${ }^{26}$ or encapsulating both PA and PDT agents within a nanoentity, such as liposomes. ${ }^{27-31}$ Specifically in the latter case, it becomes critical to evaluate the interactions between the optical dyes, such as BPD and ICG, prior to extensive exploration on the nanoformulations. Loading the nanoentities with high concentrations of BPD and ICG for better signal-to-noise ratio or higher PS delivery can lead to unstable nanoparticles, quenching, and aggregation effects. While the combination of ICG and BPD is untried in a nanoformulation, in this study, we explore for the first time the mutual effects of ICG on BPD's phototoxicity and BPD's oxidation effect on ICG's PA signal-generation capabilities. We also perform studies on two oral cancer cell lines to show the phototoxic effect of BPD when irradiated with a CW laser generally used for PDT or a nanosecond pulsed laser light that is utilized for PAI. These studies inform us that BPD and ICG combination can be utilized for PAI-guided PDT, and it points the need to consider the mutual impact when designing drug delivery strategies.

\section{Materials and Methods}

\subsection{Cell Culture}

$\mathrm{FaDu}$ (hypopharyngeal squamous cell carcinoma) and SCC4 (tongue squamous cell carcinoma) cell lines were purchased from American Type Culture Collection. FaDu and SCC4 were cultured using Eagle's minimum essential medium (EMEM) and Dulbecco's modified Eagle's medium (DMEM) media, respectively. The media was supplemented with fetal bovine serum and antibiotics. Cells were grown in T75 flasks and maintained in a standard cell culture environment.

\subsection{Photodynamic Efficacy of Benzoporphyrin Derivative in the Presence of Indocyanine Green}

Post trypsinization, cells were counted and plated on a 24-well plate at a density of 20,000 cells per well. One day later, fresh media containing BPD (Benzoporphyrin derivative monoacid ring A, Verteporfin, $718.794 \mathrm{~g} / \mathrm{mol}$, Sigma-Aldrich) and ICG (774.98 g/mol, U.S. Pharmacopeia) in varying ratios (BPD:ICG concentrations-0.25:0, 0.25:0.0625, 0.25:0.25, 0.25:0.5, 0.25:1, and $0: 1 \mu \mathrm{M}$, respectively) was added to the cells and incubated for $1 \mathrm{~h}$. The concentration and incubation period was chosen for minimal dark toxicity and maximal dye uptake based on 
previously reported studies. ${ }^{18,32-34}$ After the old media was replaced with fresh media, the cells were irradiated with a $690 \mathrm{~nm} \mathrm{CW}$ laser (HPD 7401 Laser Source) at an irradiance of $50 \mathrm{~mW} / \mathrm{cm}^{2}$ and energy densities of 1,5 , and $10 \mathrm{~J} / \mathrm{cm}^{2}$. To evaluate if PAI by itself could cause cell damage with BPD or ICG, in another set of experiments, the cells were treated with 690 and $800 \mathrm{~nm}$ pulsed laser (Optical Parameter Oscillator Laser from Opotek, Phocus BENCHTOP, 690 to $950 \mathrm{~nm}$ ) at an energy of $5 \mathrm{~mJ} /$ pulse for 200,1000, and 2000 pulses to match the irradiation time equivalent to the $\mathrm{CW}$ dose. Cell viability was calculated $24 \mathrm{~h}$ post treatment using Vybrant MTT Cell Proliferation Assay Kit (\#V13154, Thermofisher). Six experimental repeats were performed.

\subsection{Benzoporphyrin Derivative and Indocyanine Green Interactions in Solutions}

For quantifying the decrease in absorbance of ICG due to PDT, we devised an experiment where solutions of BPD and ICG at various ratios in dimethyl sulfoxide (DMSO) were irradiated with a $\mathrm{CW}$ laser at $690 \mathrm{~nm}$ or a nanosecond pulsed laser operating either at 690 or $800 \mathrm{~nm}$ wavelength. The CW laser irradiance was $50 \mathrm{~mW} / \mathrm{cm}^{2}$ and solutions received a dose of 5,10 , and $20 \mathrm{~J} / \mathrm{cm}^{2}$. The pulsed laser energy was $5 \mathrm{~mJ} /$ pulse and solutions were irradiated with 1000,2000 , and 4000 pulses to match the CW laser dose. Prior to and after the irradiation, absorption spectra of the solutions were recorded using a Synergy H1 microplate reader (BioTek).

\subsection{Photoacoustic Imaging of Tissue-Mimicking Phantoms}

To prepare tissue-mimicking phantoms, FaDu cells were incubated with EMEM media containing BPD $(1 \mathrm{~h})$ and ICG $(24 \mathrm{~h})$ in various ratios (BPD:ICG concentrations-0:5, 5:0, 5:5, and $0.25: 1 \mu \mathrm{M})$. The cells were washed with phosphate-buffered saline and collected using trypsin. For each condition, $20 \times 10^{6}$ cells were mixed with $400 \mu \mathrm{L}$ of $8 \%(w / v)$ gelatin and plated on a plain gelatin bed inside a silicone rubber spacer (13 mm diameter) in a 60-mm Petri dish. The photographs of the phantom are provided in Fig. S1 in the Supplementary Material. PAI and ultrasound (US) images were acquired using a VisualSonics LZ250 optical fiber bundle-coupled $15 \mathrm{MHz}$ ultrasound transducer (bandwidth $=13$ to $24 \mathrm{MHz}$, number of elements $=256$, and aperture $=22.5 \mathrm{~mm}$ ) connected to the Verasonics HF Vantage 256 system. A Q-switched, wavelength tunable laser operated at $10 \mathrm{~Hz}$ pulse repetition rate and 4 to $7 \mathrm{~ns}$ pulse width was used as the coherent light source. Light was delivered through a fiber-optic bundle focused at around 11-mm depth away from the transducer. The Vantage system and the Opotek laser were triggered using a Rigol DG 4102 signal generator at single polarity $5 \mathrm{~V}, 10 \mathrm{~Hz}$ square wave with $20 \%$ duty cycle (10 ms pulse width). The graphical representation of our imaging set up is shown in Fig. 1,

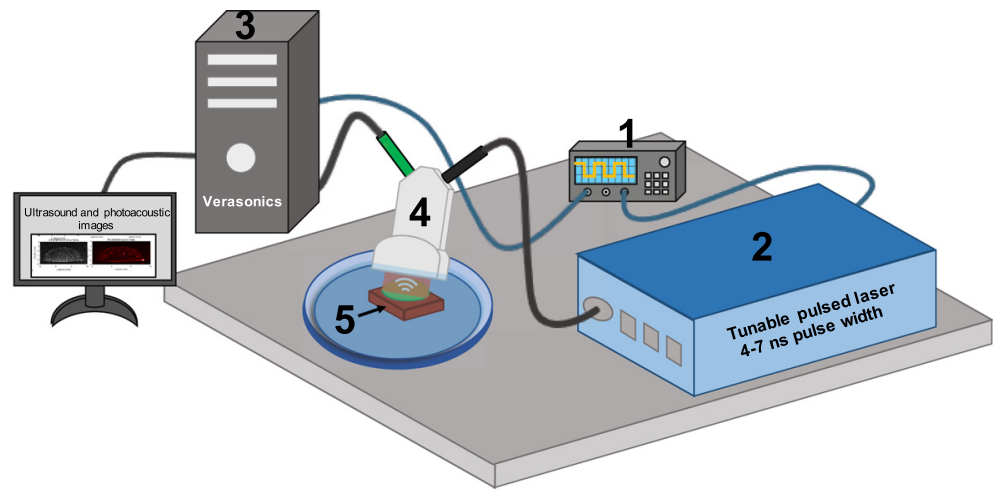

Fig. 1 Schematic representation of the imaging setup to obtain US and PA images of the tissuemimicking phantoms with a high-frequency linear array transducer. (1) Function generator, (2) tunable pulsed laser (4 to 7 ns pulse width), (3) Verasonics Vantage 256 high-frequency US platform, (4) combined US and PA transducer, and (5) tissue-mimicking phantom (photograph of the phantom provided in Fig. S1 in the Supplementary Material) 
where the tissue-mimicking sample was in the transducer imaging plane inside a Petri dish filled with deionized water for acoustic coupling between the transducer and the sample. To understand the ICG degradation in different sample combinations (with or without BPD) due to pulsed light irradiation, samples were irradiated with each wavelength $(690$ or $800 \mathrm{~nm})$ for up to $900 \mathrm{~s}$ at a rate of 10 pulses per second and $15 \mathrm{~mJ} / \mathrm{cm}^{2}$ per pulse. PA monitoring was performed at 690 or $800 \mathrm{~nm}$ at every $150 \mathrm{~s}$ intervals. All experiments were done at ambient room temperature conditions and the data were processed using customized MATLAB codes. PA images were captured at a frame rate of $10 \mathrm{~Hz}$ and the final image displayed in Figs. 6(a)-6(i) was obtained by averaging four consecutive images. The images generated had lateral and axial pixel dimensions of 88 and $44 \mu \mathrm{m}$, respectively. The PA images were compensated for the difference in laser output energy at the two wavelengths (690 and $800 \mathrm{~nm}$ ), and the background signal (captured at $900 \mathrm{~nm}$, wavelength where BPD and ICG have negligible absorption) was subtracted from the resultant image. The PA signal amplitudes from the different tissue-mimicking phantoms are displayed as a function of time in Figs. 6(j) and 6(k).

\section{Results and Discussion}

\subsection{Indocyanine Green Has Minimal Impact on the Photodynamic Efficacy of Benzoporphyrin Derivative In Vitro}

ICG has an absorption peak at around $800 \mathrm{~nm}$ with broader absorption in the 675 to $845 \mathrm{~nm}$ range at $\sim 13.5 \%$ of the peak absorbance (Fig. 2, green line). BPD has two absorption peaks, namely the Soret band at $420 \mathrm{~nm}$ and the Q band at $690 \mathrm{~nm}$, respectively (Fig. 2, red line). ICG has low absorption in the Soret band region of BPD, whereas BPD has low absorption at wavelengths greater than $720 \mathrm{~nm}$. It can be seen in Fig. 2 that both ICG and BPD have significant absorption at $690 \mathrm{~nm}$, which makes it necessary to study the interactions when they are used in combination for PAI-guided PDT.

The viability of two different cell lines (FaDu and SCC4) evaluated $24 \mathrm{~h}$ post PDT with three different types of lasers and various ratios of the two dyes is shown in Fig. 3. The BPD concentration was kept constant at $0.25 \mu \mathrm{M}$ except in the control "no-treatment" group (Fig. 3, group 1, black bars) and in the "no-BPD" group with $1 \mu \mathrm{M}$ ICG concentration (Fig. 3, group 7). The ICG concentration was $0,0.063,0.25,0.5$, and $1 \mu \mathrm{M}$ in groups 2 to 6 , respectively. The results were normalized to the control no-treatment group 1 values. The $690 \mathrm{~nm} \mathrm{CW}$ light irradiation at doses 1,5 , and $10 \mathrm{~J} / \mathrm{cm}^{2}$ in group 7 showed $99.3 \pm 9.8 \%, 100 \pm 11.4 \%$, and $102 \pm$ $8.8 \%$ of viable cells, respectively. The 690 and $800 \mathrm{~nm}$ pulsed irradiation showed cell viabilities to be $99 \pm 12.1 \%, 96 \pm 2.9 \%, 92.4 \pm 8.6 \%, 90.6 \pm 4.6 \%, 91.1 \pm 8.3 \%$, and $87.1 \pm 8 \%$ for 200 , 1000 , and 2000 pulses, respectively. Overall, there was no statistically significant difference between the control group 1 and all the light doses in group 7 for the FaDu cells (Table S1 in the Supplementary Material) at different illumination wavelengths. On the other hand,

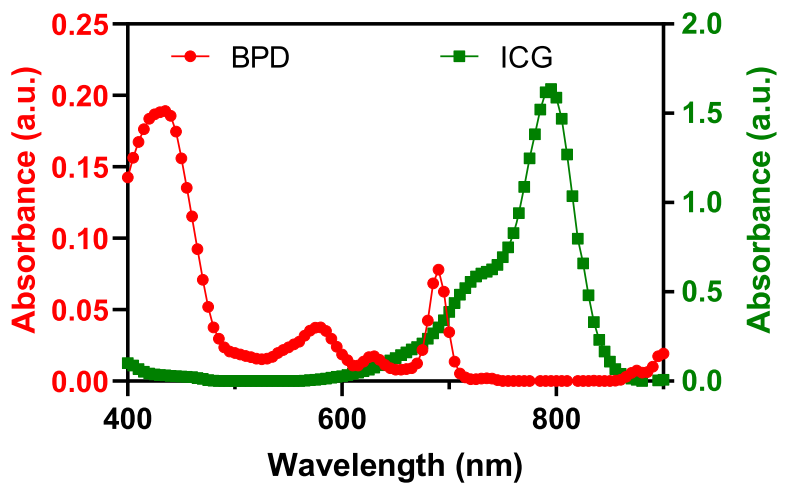

Fig. 2 Absorption spectra of BPD and ICG (5 $\mu \mathrm{M}$ concentration each). ICG has lower absorption near the Soret band of BPD, whereas BPD has lower absorption at $800 \mathrm{~nm}$. 


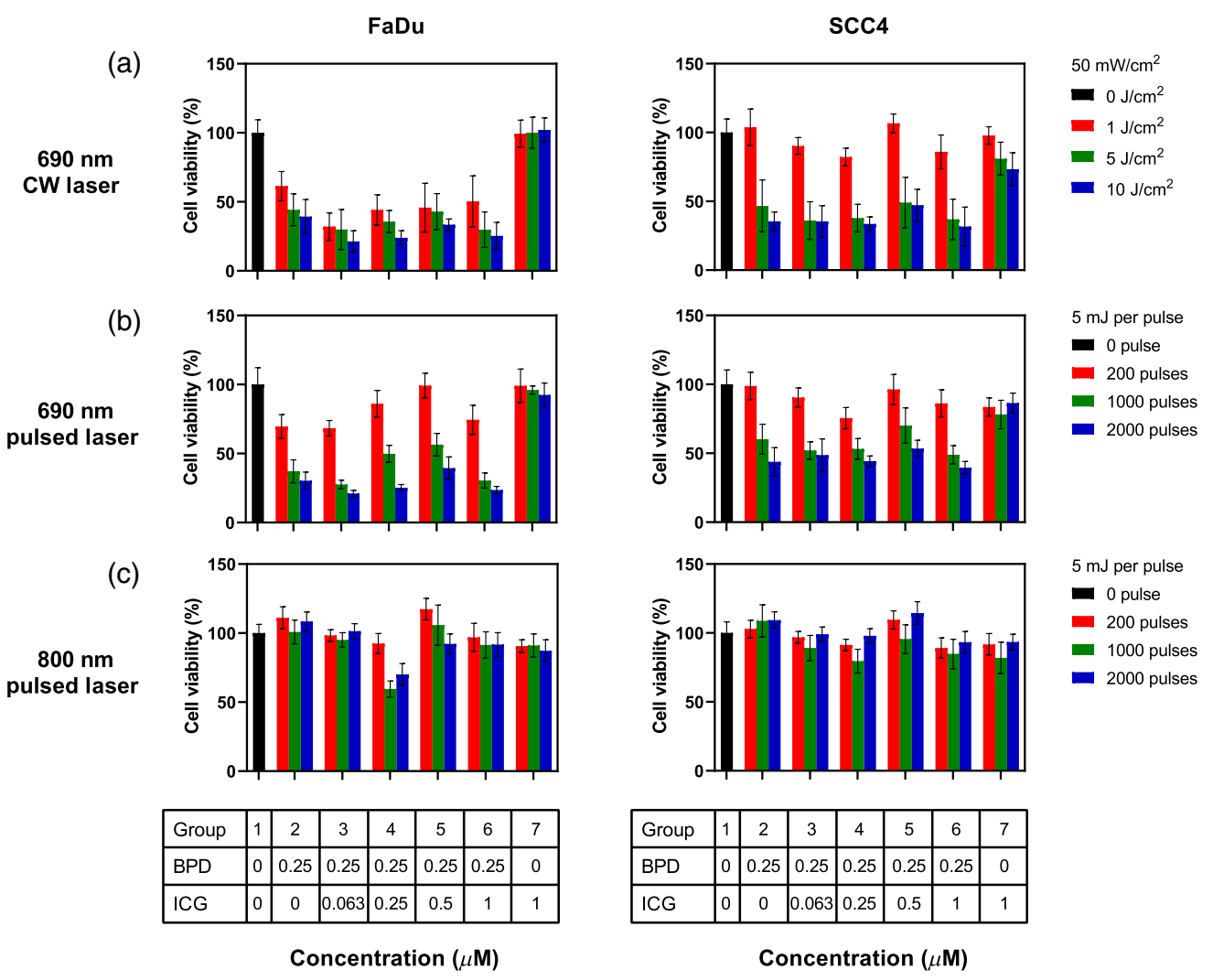

Fig. 3 Efficacy of BPD PDT in the presence or absence of ICG in two cell lines (FaDu and SCC4). Response to PDT with (a) 690-nm CW laser, (b) 690-nm pulsed laser, and (c) 800-nm pulsed laser. PDT with $690 \mathrm{~nm}$ laser (CW or pulsed) gave a good treatment response compared to $800-\mathrm{nm}$ pulsed laser. BPD's PDT efficacy remained the same in the presence of varying concentrations of ICG. Error bars represent standard error of the mean (SEM) with a total of $N=6$ experimental repeats. One-way analysis of variance (ANOVA) Dunnett's multiple comparison test was performed to identify statistically significant difference between the groups and $p$-value $<0.01$ was considered significant (Tables S1 and S2 in the Supplementary Material).

SCC4 cells treated with ICG only [Fig. 3(a), right panel, group 7] showed very minimal response at higher light dose of $10 \mathrm{~J} / \mathrm{cm}^{2}$ with $690 \mathrm{~nm} \mathrm{CW}$ laser but had no statistically significant difference at a lower doses of 1 and $5 \mathrm{~J} / \mathrm{cm}^{2}$, respectively (Table S2 in the Supplementary Material). We attribute this observation to ICG's photosensitizing properties at higher light doses. For example, to achieve significant cell death, Abels et al. ${ }^{33}$ utilized longer incubation time $(24 \mathrm{~h})$, higher ICG concentration $(>5 \mu \mathrm{M})$, and higher light dose at $800 \mathrm{~nm}\left(80 \mathrm{~mW} / \mathrm{cm}^{2}\right.$, $\left.24 \mathrm{~J} / \mathrm{cm}^{2}\right)$ than the parameters used in our study $\left(1 \mu \mathrm{M}, 1 \mathrm{~h}\right.$ accumulation, $50 \mathrm{~mW} / \mathrm{cm}^{2}$, $10 \mathrm{~J} / \mathrm{cm}^{2}$ ). We also do not see ICG being phototoxic to both FaDu and SCC4 cells for pulsed irradiation at both wavelengths for all doses (Tables S1 and S2 in the Supplementary Material). The ICG concentration used in our study for PAI did not kill the cells, but it was high enough to give us a detectable PA signal.

\subsection{Less Than $1 \mu \mathrm{M}$ Indocyanine Green Concentration Had Minimal Impact on the Photodynamic Efficacy of Benzoporphyrin Derivative In Vitro for Both Pulsed and Continuous Wave Regimes}

To check if various ICG concentrations were influencing the PDT outcome at different illumination schemes, we compared group 1 (control, no-treatment, no dye) with groups 2 to 6 . FaDu cells at $690 \mathrm{~nm} \mathrm{CW}$ illumination at 1,5 , and $10 \mathrm{~J} / \mathrm{cm}^{2}$ showed a significant difference between nontreated (group 1) and all treated groups (groups 2 to 6) containing BPD (Table S1 in the 
(a)

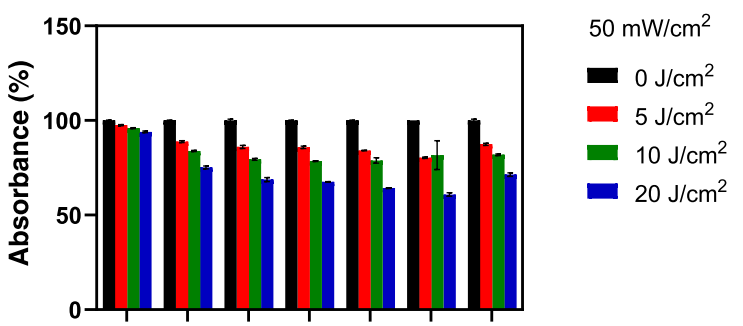

(b)

$690 \mathrm{~nm}$ pulsed laser

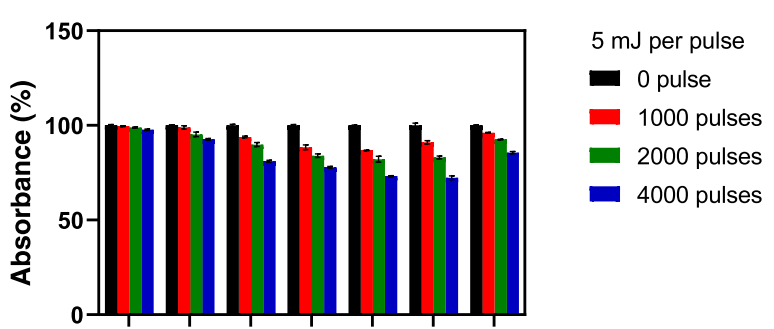

(c)

$800 \mathrm{~nm}$
pulsed laser

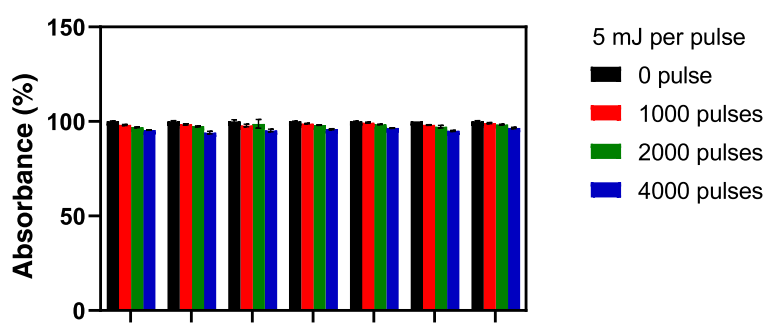

\begin{tabular}{|l|c|c|c|c|c|c|c|}
\hline Group & 1 & 2 & 3 & 4 & 5 & 6 & 7 \\
\hline BPD & 0 & 1.25 & 5 & 10 & 20 & 5 & 5 \\
\hline ICG & 5 & 5 & 5 & 5 & 5 & 1.25 & 10 \\
\hline
\end{tabular}

Concentration $(\mu \mathrm{M})$

Fig. 4 Bar graphs representing percentage decrease in absorbance of ICG at $780 \mathrm{~nm}$. The solutions were irradiated with (a) CW laser at $690 \mathrm{~nm}$ and nanosecond pulsed laser at (b) $690 \mathrm{~nm}$ and (c) $800 \mathrm{~nm}$, respectively. The error bars are SEM. Two-way ANOVA Tukey's multiple comparison test was performed to identify statistically significant difference between the different conditions and $p$-value $<0.05$ was considered significant (see Table S3 for constant ICG concentration and Table S4 for constant BPD concentration).

Supplementary Material). The same trend was observed for FaDu cells irradiated with 1000 and 2000 pulses at $690 \mathrm{~nm}$. Low number of PA pulses (200 pulses at $690 \mathrm{~nm}$ pulsed irradiation) showed minimal or nonsignificant difference compared to the control group. SCC4 cells also had similar trends, except at $1 \mathrm{~J} / \mathrm{cm}^{2}$ low-dose group CW $690 \mathrm{~nm}$ irradiation (Fig. 4, top-right panel, red bars). SCC4 and FaDu cells display different chemoresistance, ${ }^{35}$ and it is not surprising for these cells to display different PDT responses and the mechanisms involved are beyond the scope of this work and need to be studied further.

At high light doses, groups 2 to 6 showed no statistical difference between $690 \mathrm{~nm} \mathrm{CW}$ or pulsed laser irradiation at both 690 and $800 \mathrm{~nm}$ (Tables S1 and S2 in the Supplementary Material). At lower light dose, pulsed irradiation caused less cell death than CW laser (Fig. 3, red bars, top and middle panel). These observations are in congruence with Pogue et al. ${ }^{25}$ and Kawauchi et al. ${ }^{36}$ Particularly for lower doses, the differences were observed, because pulsed PDT process consumes oxygen at a lower rate compared to CW laser, impacting singlet oxygen generation and therefore less therapeutic efficacy. Based on these observations and values, for the light doses and concentrations (below $1 \mu \mathrm{M}$ ) used, ICG did not influence BPDPDT response. 


\subsection{Photoacoustic Monitoring of Indocyanine Green Photodegradation in the Presence of Benzoporphyrin Derivative}

PA signal intensity is highly dependent on the optical absorption coefficient and concentration of the dye being imaged. ${ }^{13,16}$ It has been shown previously that ICG, a tricarbocyanine dye, decomposes with light ( 20 pulses of $10 \mathrm{~ms}$ duration and total energy of $50 \mathrm{~J})^{37}$ and undergoes selfsensitized photodegradation. To evaluate if such an effect exists on ICG due to PA pulsed laser in the presence or absence of BPD, we performed experiments in solution and in tissue-mimicking phantoms.

The change in ICG absorbance $780 \mathrm{~nm}$ post irradiation with CW laser at $690 \mathrm{~nm}$ and nanosecond pulsed laser at 690 or $800 \mathrm{~nm}$ is shown in Fig. 4. Irradiation was performed on various solutions with varying concentrations of BPD (0 to $20 \mu \mathrm{M})$ and ICG $(1.25$ to $10 \mu \mathrm{M})$. BPD has low absorbance at $780 \mathrm{~nm}$ (Fig. 2) and hence the condition BPD: ICG 5: $0 \mu \mathrm{M}$ is not included in these data. Irradiation at $800 \mathrm{~nm}$ caused minimal ICG photodegradation (less than 5\% decrease in absorbance), irrespective of the BPD concentration and light dose [Fig. 4(c)]. A significant difference was observed only between the no-treatment group [Fig. 4(c), black bars] and highest light dose [Fig. 4(c), 4000 pulses, blue bars]. A greater degree of ICG photodegradation was observed for both CW and pulsed $690 \mathrm{~nm}$ irradiation, compared to $800 \mathrm{~nm}$ pulsed irradiation. The extent of photodegradation was maximum for higher light doses. In addition, at $690 \mathrm{~nm}$ irradiation, CW laser produced more ICG photodegradation than the pulsed laser. The ICG photodegradation increased with increase in BPD concentration in the solution (Fig. 4, groups 1 to 5). On the other hand, ICG photodegradation decreased with an increase in ICG concentration (Fig. 4, groups 3, 6, and 7). Similar trend (decrease in absorbance) was observed for ICG's absorbance at $690 \mathrm{~nm}$ (Fig. S2 in the Supplementary Material), although the percentage decrease was lesser than $780 \mathrm{~nm}$ absorbance as expected.

Next, we irradiated the mixture of BPD and ICG solutions in the presence and absence of sodium azide $\left(\mathrm{NaN}_{3}\right)$, a quencher of singlet oxygen, to reinforce our observations in Fig. 4 . The percentage decrease in absorbance at $780 \mathrm{~nm}$ from three different BPD:ICG ratio solutions is demonstrated in Fig. 5. We observed $\sim 30 \%$ less decrease in absorbance in the presence of $\mathrm{NaN}_{3}$ (Fig. 5, black bars) than in the absence of $\mathrm{NaN}_{3}$ (Fig. 5, red bars). Irrespective of the BPD:ICG ratio, we observe that $\mathrm{NaN}_{3}$ attracts the BPD-generated singlet oxygen molecules before they can oxidize ICG.

Encouraged by the outcomes in Figs. 4 and 5, experiments were performed to determine if similar trends can be monitored with PAI in a tissue-mimicking environment. Phantoms containing FaDu cells incubated in either BPD, ICG, or a mixture (Fig. 6) underwent PAI at wavelengths 690, 800, and $900 \mathrm{~nm}$, respectively. The PA images were plotted along with their US counterparts (Fig. 6, grayscale images, right panel) to facilitate comparison with the structural shape of the tissue-mimicking phantoms. As expected, the sample containing BPD alone had

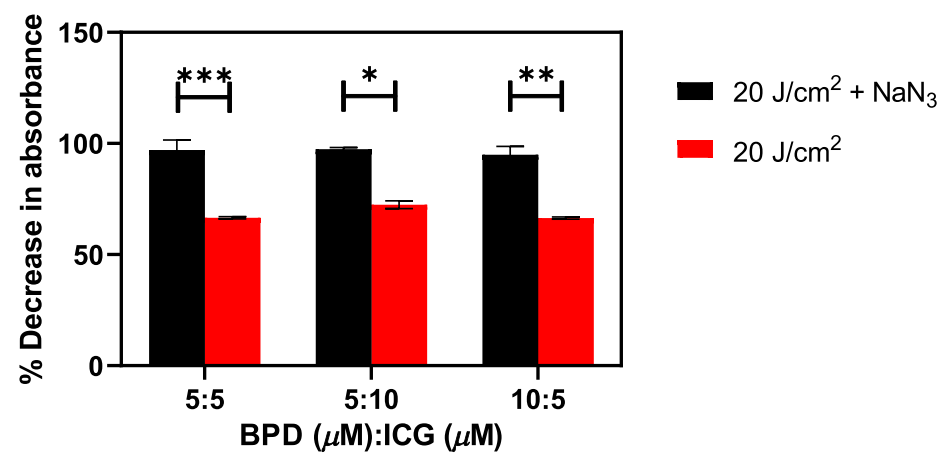

Fig. 5 Bar graphs depicting percentage decrease in absorbance of the BPD and ICG mixture at varying concentrations in the presence (black bars) and absence (red bars) of $\mathrm{NaN}_{3}$ when treated with $690 \mathrm{~nm}$ CW laser. The error bars are SEM. Two-way ANOVA Tukey's multiple comparison test was performed to identify statistically significant difference between the different conditions and $p$-value $<0.05$ was considered significant. 

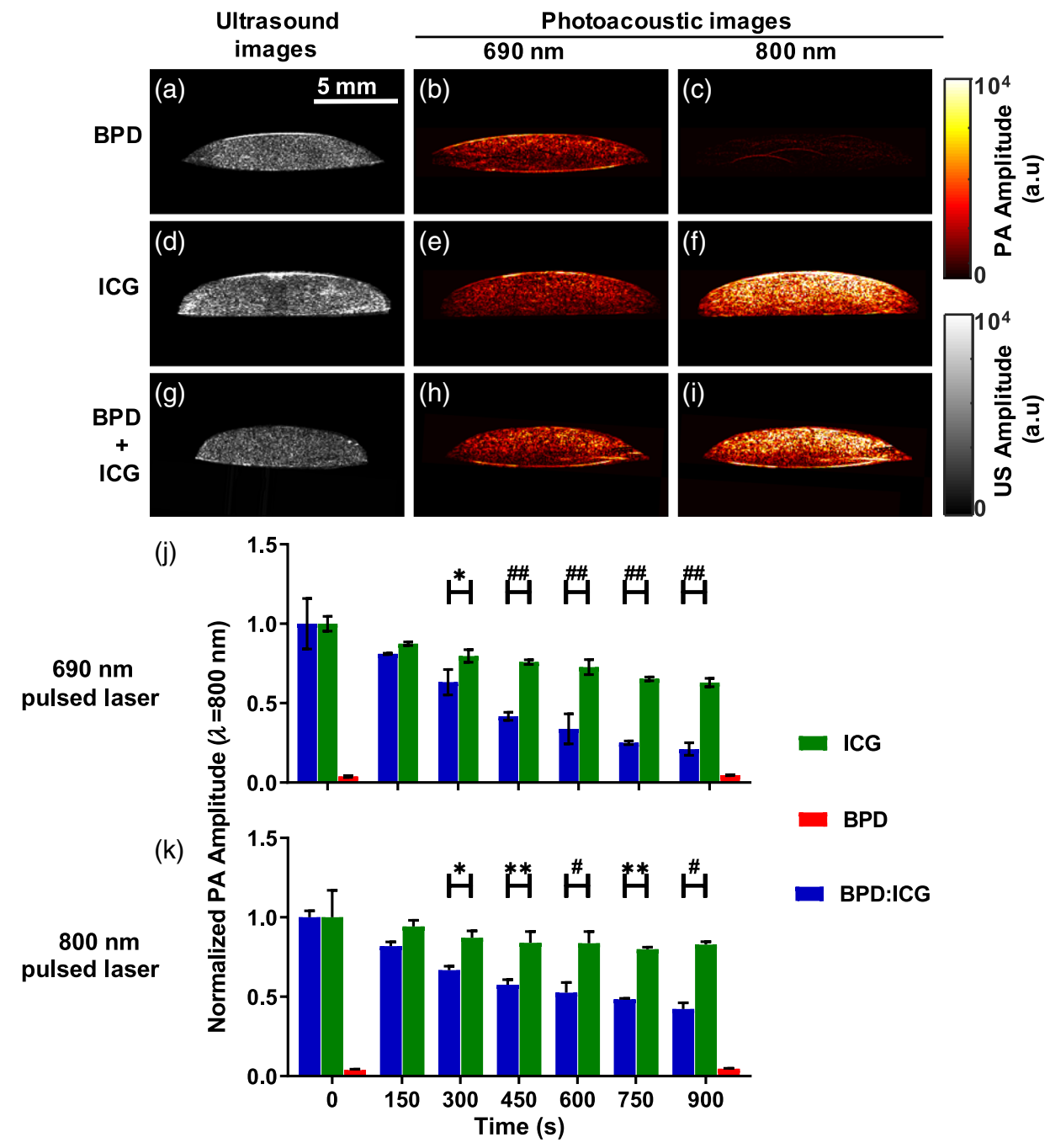

Fig. 6 (a), (d), and (g) US and PA images at (b), (e), and (h) $690 \mathrm{~nm}$ and (c), (f), and (i) $800 \mathrm{~nm}$ of a tissue-mimicking phantom made of FaDu cells incubated with (a)-(c) $5 \mu \mathrm{M}$ BPD (for $1 \mathrm{~h}$ ), (d)-(f) $5 \mu \mathrm{M}$ ICG (for $24 \mathrm{~h}$ ), and (g)-(i) BPD and ICG combination for 1 and $24 \mathrm{~h}$, respectively. (j) and (k) Normalized PA signal as a function of pulsed irradiation time (or PDT power), monitored at $800 \mathrm{~nm}$; (j) irradiation at $690 \mathrm{~nm}$ for ICG $5 \mu \mathrm{M}$ (green), BPD $5 \mu \mathrm{M}$ (red), and BPD: ICG $=5: 5 \mu \mathrm{M}$ (blue); (k) similar to (j) except with $800 \mathrm{~nm}$ irradiation. Error bars show the standard deviations from three separate measurements taken at each interval. BPD-only signal (red) was close to the noise level because of negligible absorption at $800 \mathrm{~nm}$. Two-way ANOVA Sidak's multiple comparison test was performed to identify statistically significant difference between the groups and $p$-value, where $<0.05$ was considered significant; ${ }^{*} p<0.05,{ }^{* *} p<0.01,{ }^{\#} p<0.001$, and ${ }^{\# \#} p<0.0001$.

higher PA signal at $690 \mathrm{~nm}$ than at $800 \mathrm{~nm}$ [Figs. 6(b) and 6(c)]. The sample with ICG alone had higher PA signal at $800 \mathrm{~nm}$ than at $690 \mathrm{~nm}$ [Figs. 6(e) and 6(f)]. In agreement with absorbance curves shown in Fig. 2, the sample with both BPD and ICG had higher PA signal at $800 \mathrm{~nm}$ than at $690 \mathrm{~nm}$ [Figs. 6(h) and 6(i)] as molar absorption coefficient of ICG is greater than BPD. ${ }^{38,39}$ Next, we irradiated the sample with pulsed laser $(690$ or $800 \mathrm{~nm})$ for $\sim 900$ s to photoacoustically monitor ICG's degradation. We observed the PA signal intensity decreases with pulsed laser exposure time as shown in Figs. 6(j) and 6(k). The presence of BPD [Figs. 6(j) and 6(k), blue bars] enhanced the ICG photodegradation in the mixture sample, i.e., the decrease in PA signal intensity was higher than in the sample with ICG alone [Figs. 6(j) and 6(k), green bars] and these differences were statistically significant. Furthermore, as expected from Figs. 4 and 5, the photodegradation with $690 \mathrm{~nm}$ pulsed irradiation is higher than $800 \mathrm{~nm}$ pulsed irradiation. These results clearly demonstrate that PAI can be used to monitor ICG photodegradation in the presence of singlet oxygen-generating molecules such as BPD. 
BPD and ICG have molar extinction coefficient of $34,895 \mathrm{M}^{-1} \mathrm{~cm}^{-1}$ at $687 \mathrm{~nm}$ in DMSO 39 and $113790 \mathrm{M}^{-1} \mathrm{~cm}^{-1}$ at $780 \mathrm{~nm}$ in water, ${ }^{38}$ respectively. Both the dyes have significantly higher absorption and can be distinguished from the endogenous chromophores such as hemoglobin via fluorescence ${ }^{40,41}$ or PAI. ${ }^{13,42-45}$ BPD is an excellent PS, whereas ICG needs very high PDT dose $\left(\mu \mathrm{M} \times \mathrm{J} / \mathrm{cm}^{2}\right)$ due to its low singlet oxygen quantum yield. More than a regular PS behavior, ICG molecule relaxes via nonradiative mechanisms, such as heat, and hence is a very good PA or photothermal therapy agent. ${ }^{22}$ ICG is an interesting molecule with properties that heavily depend on the concentration and the surrounding environment. ${ }^{38,46}$ Several studies have shown that ICG demonstrates a blueshift in absorbance peak with an increase in the concentration or in the presence of serum, water, or salt, etc..$^{38,46-49}$ and degrades when irradiated with light. ${ }^{50}$ Specifically, ICG undergoes conformational changes in the presence of reactive radicals that can either be self-generated or be generated by other molecules in the vicinity. J-aggregated ICG molecules or conjugation to plasmonic nanoparticles are shown to enhance ICG photostability. ${ }^{51-53}$ The photodegradation properties of ICG are also cleverly utilized for drug delivery strategies as reviewed recently by Gorka and Schnermann. ${ }^{51}$ Furthermore, ICG was shown to have enhanced photostability via encapsulation in lipids or association with plasmonic gold colloids. ${ }^{54-56}$ All these studies clearly point out the versatility of ICG as a theranostic agent and the need for thorough photostability characterization in the presence of other dyes or oxidizing agents in these formulations both in solutions and in cellular environment.

\section{Conclusions}

Here we evaluated the mutual impact of BPD and ICG on their PDT efficacy and PA signal stability. We observed no effect of ICG on the phototoxic capability of BPD at both 690- and 800-nm laser illumination. We also did not notice significant difference in BPD phototoxicity between $\mathrm{CW}$ and pulsed laser illumination at $690 \mathrm{~nm}$ in the presence of ICG. We, however, observed that the presence of BPD enhanced photodegradation of ICG, leading to a reduction in optical absorption and corresponding decrease in PA signal intensity at $800 \mathrm{~nm}$. Thus far, we are the first group to study the interactions between BPD and ICG for both continuous and pulsed illumination. As BPD is primarily a type II PS that generates reactive oxygen species, it remains to be seen if ICG would react in a similar fashion in the presence of a type I PS that generates other reactive molecular species. Overall, the photointeractions between the two dyes in close proximity emphasizes the need to incorporate such studies prior to the design of multiagent nanoentities. Our future studies in tissue-mimicking phantoms and preclinical cancer models will involve incorporation of these dyes within nanoentities to shield one another from the oxidation effects observed here and further enhance the image contrast while preserving the PDT efficacy.

\section{Disclosures}

The authors have no financial conflicts of interest to disclose.

\section{Acknowledgments}

The authors gratefully acknowledge the funds from NIH R01CA231606, R41CA221420, EY028839, and School of Engineering, Tufts University. The authors would also like to thank Dr. Kimberly Homan and Dr. Jason Cook for their insightful discussions.

\section{References}

1. M. Triesscheijn et al., "Photodynamic therapy in oncology," Oncologist 11, 1034-1044 (2006).

2. P. Agostinis et al., "Photodynamic therapy of cancer: an update," CA Cancer J. Clin. 61, 250-281 (2011).

3. D. E. Dolmans, D. Fukumura, and R. K. Jain, "Photodynamic therapy for cancer," Nat. Rev. Cancer 3, 380-387 (2003). 
4. Z. Huang et al., "Photodynamic therapy for treatment of solid tumors-potential and technical challenges," Technol. Cancer Res. Treat. 7, 309-320 (2008).

5. S. Mallidi, B. Q. Spring, and T. Hasan, "Optical imaging, photodynamic therapy and optically triggered combination treatments," Cancer J. 21, 194-205 (2015).

6. Z. Huang, "A review of progress in clinical photodynamic therapy," Technol. Cancer Res. Treat. 4, 283-293 (2005).

7. J. P. Celli et al., "Imaging and photodynamic therapy: mechanisms, monitoring, and optimization," Chem. Rev. 110, 2795-2838 (2010).

8. S. Mallidi et al., "Platform for ergonomic intraoral photodynamic therapy using low-cost, modular 3D-printed components: design, comfort and clinical evaluation," Sci. Rep. 9, 15830 (2019).

9. B. W. Pogue et al., "Revisiting photodynamic therapy dosimetry: reductionist \& surrogate approaches to facilitate clinical success," Phys. Med. Biol. 61, R57-R89 (2016).

10. B. C. Wilson, M. S. Patterson, and L. Lilge, "Implicit and explicit dosimetry in photodynamic therapy: a new paradigm," Lasers Med. Sci. 12, 182-199 (1997).

11. X. Zhou et al., "Pretreatment photosensitizer dosimetry reduces variation in tumor response," Int. J. Radiat. Oncol. Biol. Phys. 64, 1211-1220 (2006).

12. S. Mallidi et al., "Photosensitizer fluorescence and singlet oxygen luminescence as dosimetric predictors of topical 5-aminolevulinic acid photodynamic therapy induced clinical erythema," J. Biomed. Opt. 19, 028001 (2014).

13. S. Mallidi, G. P. Luke, and S. Emelianov, "Photoacoustic imaging in cancer detection, diagnosis, and treatment guidance," Trends Biotechnol. 29, 213-221 (2011).

14. J. Xia, J. Yao, and L. Wang, "Photoacoustic tomography: principles and advances," Electromagn. Waves 147, 1-22 (2014).

15. M. Li, Y. Tang, and J. Yao, "Photoacoustic tomography of blood oxygenation: a mini review," Photoacoustics 10, 65-73 (2018).

16. M. Xu and L. V. Wang, "Photoacoustic imaging in biomedicine," Rev. Sci. Instrum. 77, 041101 (2006).

17. C. J. Ho et al., "Multifunctional photosensitizer-based contrast agents for photoacoustic imaging," Sci. Rep. 4, 5342 (2014).

18. G. Obaid et al., "Impacting pancreatic cancer therapy in heterotypic in vitro organoids and in vivo tumors with specificity-tuned, NIR-activable photoimmunonanoconjugates: towards conquering desmoplasia?" Nano Lett. 19, 7573-7587 (2019).

19. J. Wang et al., "Switchable photoacoustic intensity of methylene blue via sodium dodecyl sulfate micellization," Langmuir 34, 359-365 (2018).

20. J. Wang et al., "A mechanistic investigation of methylene blue and heparin interactions and their photoacoustic enhancement," Bioconjug. Chem. 29, 3768-3775 (2018).

21. H. Wang et al., "Indocyanine green-incorporating nanoparticles for cancer theranostics," Theranostics 8, 1227-1242 (2018).

22. C. Giraudeau et al., "Indocyanine green: photosensitizer or chromophore? Still a debate," Curr. Med. Chem. 21, 1871-1897 (2014).

23. S. Reindl et al., "Quantum yield of triplet formation for indocyanine green," J. Photochem. Photobiol. A 105, 65-68 (1997).

24. A. D. Tekrony et al., "Photobleaching kinetics of Verteporfin and Lemuteporfin in cells and optically trapped multilamellar vesicles using two-photon excitation," Photochem. Photobiol. 87, 853-861 (2011).

25. B. W. Pogue et al., "Absorbed photodynamic dose from pulsed versus continuous wave light examined with tissue-simulating dosimeters," Appl. Opt. 36, 7257-7214 (1997).

26. J. Lin et al., "Photosensitizer-loaded gold vesicles with strong plasmonic coupling effect for imaging-guided photothermal/photodynamic therapy," ACS Nano 7, 5320-5329 (2013).

27. H. Xu et al., "PEGylated liposomal photosensitizers as theranostic agents for dual-modal photoacoustic and fluorescence imaging-guided photodynamic therapy," J. Innov. Opt. Health Sci. 12(3), 1941003 (2019).

28. J. E. Lemaster and J. V. Jokerst, "What is new in nanoparticle-based photoacoustic imaging?" WIREs Nanomed. Nanobiotechnol. 9 (2017). 
29. Y. Zhang and J. F. Lovell, "Recent applications of phthalocyanines and naphthalocyanines for imaging and therapy," WIREs Nanomed. Nanobiotechnol. 9 (2016).

30. G. Obaid et al., "Photonanomedicine: a convergence of photodynamic therapy and nanotechnology," Nanoscale 8, 12471-12503 (2016).

31. P. Rai et al., "Development and applications of photo-triggered theranostic agents," $A d v$. Drug Delivery Rev. 62, 1094-1124 (2010).

32. H. C. Huang et al., "Photodynamic therapy synergizes with irinotecan to overcome compensatory mechanisms and improve treatment outcomes in pancreatic cancer," Cancer Res. 76, 1066-1077 (2016).

33. C. Abels et al., "Indocyanine green (ICG) and laser irradiation induce photooxidation," Arch. Dermatol. Res. 292, 404-411 (2000).

34. M. D. Savellano and T. Hasan, "Photochemical targeting of epidermal growth factor receptor: a mechanistic study," Clin. Cancer Res. 11, 1658-1668 (2005).

35. K. J. Cullen et al., "Glutathione S-transferase $\pi$ amplification is associated with cisplatin resistance in head and neck squamous cell carcinoma cell lines and primary tumors," Cancer Res. 63, 8097-8102 (2003).

36. S. Kawauchi et al., "Differences between cytotoxicity in photodynamic therapy using a pulsed laser and a continuous wave laser: study of oxygen consumption and photobleaching," Lasers Med. Sci. 18, 179-183 (2004).

37. E. Engel et al., "Light-induced decomposition of indocyanine green," Invest. Ophthalmol. Visual Sci. 49, 1777-1783 (2008).

38. M. L. Landsman et al., "Light-absorbing properties, stability, and spectral stabilization of indocyanine green," J. Appl. Physiol. 40, 575-583 (1976).

39. B. Aveline, T. Hasan, and R. W. Redmond, "Photophysical and photosensitizing properties of benzoporphyrin derivative monoacid ring A (BPD-MA)," Photochem. Photobiol. 59, 328-335 (1994).

40. J. C. Kraft and R. J. Y. Ho, "Interactions of indocyanine green and lipid in enhancing nearinfrared fluorescence properties: the basis for near-infrared imaging in vivo," Biochemistry 53, 1275-1283 (2014).

41. S. Mordon et al., "Indocyanine green: physicochemical factors affecting its fluorescence in vivo," Microvasc. Res. 55, 146-152 (1998).

42. M. D. Laramie et al., "Small molecule optoacoustic contrast agents: an unexplored avenue for enhancing in vivo imaging," Molecules 23, 2766 (2018).

43. G. P. Luke, D. Yeager, and S. Y. Emelianov, "Biomedical applications of photoacoustic imaging with exogenous contrast agents," Ann. Biomed. Eng. 40, $422-437$ (2012).

44. J. Weber, P. C. Beard, and S. E. Bohndiek, "Contrast agents for molecular photoacoustic imaging," Nat. Methods 13, 639-650 (2016).

45. Q. Fu et al., "Photoacoustic imaging: contrast agents and their biomedical applications," Adv. Mater. 31, 1805875 (2019).

46. W. Holzer et al., "Photostability and thermal stability of indocyanine green," J. Photochem. Photobiol. B 47, 155-164 (1998).

47. B. H. Yuan, N. G. Chen, and Q. Zhu, "Emission and absorption properties of indocyanine green in Intralipid solution," J. Biomed. Opt. 9, 497-503 (2004).

48. S. Mindt et al., "Stability and degradation of indocyanine green in plasma, aqueous solution and whole blood," Photochem. Photobiol. Sci. 17, 1189-1196 (2018).

49. A. K. Kirchherr, A. Briel, and K. Mader, "Stabilization of indocyanine green by encapsulation within micellar systems," Mol. Pharmaceutics 6, 480-491 (2009).

50. E. Engel et al., "Light-induced decomposition of indocyanine green," Invest. Ophthalmol. Visual Sci. 49, 1777-1783 (2008).

51. A. P. Gorka and M. J. Schnermann, "Harnessing cyanine photooxidation: from slowing photobleaching to near-IR uncaging," Curr. Opin. Chem. Biol. 33, 117-125 (2016).

52. F. M. Penha et al., "Biochemical analysis and decomposition products of indocyanine green in relation to solvents, dye concentrations and laser exposure," Ophthalmologica 230, 59-67 (2013).

53. J. Chen et al., "Indocyanine green-loaded gold nanostars for sensitive SERS imaging and subcellular monitoring of photothermal therapy," Nanoscale 9, 11888-11901 (2017). 
Petrovic et al.: Mutual impact of clinically translatable near-infrared dyes on photoacoustic image contrast...

54. C. D. Geddes, H. S. Cao, and J. R. Lakowicz, "Enhanced photostability of ICG in close proximity to gold colloids," Spectrochim. Acta, Part A 59, 2611-2617 (2003).

55. A. Yuan et al., "Activatable photodynamic destruction of cancer cells by NIR dye/ photosensitizer loaded liposomes," Chem. Commun. 51, 3340-3342 (2015).

56. D. Miranda et al., "Indocyanine green binds to DOTAP liposomes for enhanced optical properties and tumor photoablation," Biomater. Sci. 7, 3158-3164 (2019).

Biographies of the authors are not available. 